\title{
Norm Convergence in the Space of Hyper-functions
}

\author{
A. N. Deepthi ${ }^{1}$ and N. R. Mangalambal ${ }^{2}$ \\ ${ }^{1}$ Department of Mathematics, Sree Narayana College, Nattika, Thrissur, Kerala \\ ${ }^{1 \& 2}$ Centre for Research in Mathematical Sciences, St. Joseph's College (Autonomous), Irinjalakuda, Kerala, India \\ E-Mail: deepthiakshaya@gmail.com, thottuvai@gmail.com
}

\begin{abstract}
Classical Mathematics is not sufficient to justify some functions like Dirac's delta function, Heaviside's unit step function in the mathematical modelling of some physical problems. Mikio Sato introduced the concept of hyperfunction to explain such situations. He gave a new generalisation to such functions using the theory of complex analysis. Hyperfunctions have many applications in the field of differential equations that are related with the physical problems involving Heat equation, wave equation etc. Urs Graf applied various transforms to hyperfunctions. With the help of these transforms he solved differential equations in terms of hyperfunctions. In this paper we defined a norm to a subclass of the linear space of hyperfunctions. The completeness and separability properties of this subfamily of hyperfunctions are established in this paper. Hyperfunctions of bounded exponential growth with compact support are mainly considering here.We have developed the results using the defining function of the hyperfunction. Hence we give a normed space approach to the subfamily of hyperfunctions having bounded exponential growth with compact support. Mathematics Subject Classification: 46F15, 46BXX

Keywords: Hyper Functions, Normed Spaces, Banach Spaces
\end{abstract}

\section{INTRODUCTION}

Mikio Sato introduces the idea of hyper-functions to mention his generalization of the concept of functions [5],[6],[7]. Stevan Pilipovic and Bogoljub Stankovic discussed the convergence in the space of Fourier hyperfunctionsin their paper [2].The concept of measurable [4] hyper-function is introduced in this paper and a norm [3] is defined to a subfamily of hyper-functionshaving bounded exponential growth with compact support. We defined the convergence in that subspace of hyper-function in terms of the new defined norm. We have established some properties of this convergence using the defining function of such hyper-functions.

\section{PRELIMINARIES}

We denote the upper and lower half-plane of the complex plane $C$ by $C_{+}=\{z \in C: I z>0\}, C=\{z \in C: I z<0\}$ respectively.

Definition 1[1]:For an open interval I of the real line, the open subset $\mathrm{N}(\mathrm{I})$ subset of $\mathrm{C}$ is called a complex neighbourhood of I, if I is a closed subset of $\mathrm{N}(\mathrm{I})$.

Let $\mathrm{N}_{+}(\mathrm{I})=\mathrm{N}(\mathrm{I}) \cap \mathrm{C}_{+}$and $\mathrm{N}_{-}(\mathrm{I})=\mathrm{N}(\mathrm{I}) \cap \mathrm{C}$.

Let $\mathrm{O}(\mathrm{N}(\mathrm{I}) \backslash \mathrm{I})$ denotes the ring of holomorphic functions in $\mathrm{N}(\mathrm{I}) \backslash \mathrm{I}$.
For a given interval I a function $\mathrm{F}(\mathrm{z}) \in \mathrm{O}(\mathrm{N}(\mathrm{I}) \backslash \mathrm{I})$ can be written as

$F(z)=\left\{\begin{array}{l}F_{+}(z) \text { for } z \in N_{+}(I) \\ F_{-}(\mathrm{z}) \text { for } z \in N_{-}(I)\end{array}\right.$

whereF $_{+}(\mathrm{z}) \in \mathrm{O}\left(\mathrm{N}_{+}(\mathrm{I})\right)$ and $\mathrm{F}_{-}(\mathrm{z}) \in \mathrm{O}\left(\mathrm{N}_{-}(\mathrm{I})\right)$, are called upper and lower component of $\mathrm{F}(\mathrm{z})$ respectively. In general the upper and lower component of $F(z)$ need not be related to each other. If they are analytic continuations from each other, we call $\mathrm{F}(\mathrm{z})$ a global analytic function on $\mathrm{N}(\mathrm{I})$ and we can write $\mathrm{F}_{+}(\mathrm{z})=\mathrm{F} .(\mathrm{z})=\mathrm{F}(\mathrm{z})$

Definition 2[1]: Two functions $\mathrm{F}(\mathrm{z})$ and $\mathrm{G}(\mathrm{z})$ in $\mathrm{O}(\mathrm{N}(\mathrm{I}) \backslash \mathrm{I})$ are equivalent if for $\mathrm{z} \in \mathrm{N}_{1}(\mathrm{I}) \cap \mathrm{N}_{2}(\mathrm{I}), \mathrm{G}(\mathrm{z})=\mathrm{F}(\mathrm{z})+\varphi(\mathrm{z})$, with $\varphi(\mathrm{z}) \in \mathrm{O}(\mathrm{N}(\mathrm{I}))$ where $\mathrm{N}_{1}(\mathrm{I})$ and $\mathrm{N}_{2}(\mathrm{I})$ are complex neighbourhoods of $\mathrm{I}$ of $\mathrm{F}(\mathrm{z})$ and $\mathrm{G}(\mathrm{z})$ respectively.

Definition 3[1]: An equivalence class of functions $\mathrm{F}(\mathrm{z}) \epsilon$ $\mathrm{O}(\mathrm{N}(\mathrm{I}) \backslash \mathrm{I})$ defines a hyper-function $\mathrm{f}(\mathrm{x})$ on $\mathrm{I}$. Which is denoted by $f(x)=[F(z)]=\left[F_{+}(z), F_{-}(z)\right] . F(z)$ is called defining or generating function of the hyper-function.

The set of all hyper-functions defined on the interval $\mathrm{I}$ is denoted by $\mathrm{B}(\mathrm{I})$.

$$
\mathrm{B}(\mathrm{I})=\mathrm{O}(\mathrm{N}(\mathrm{I}) \backslash \mathrm{I}) \backslash \mathrm{O}(\mathrm{N}(\mathrm{I}))
$$

A real analytic function $\varphi(x)$ on I is defined by the fact that $\varphi(x)$ can analytically be continued to a full neighbourhood $\mathrm{U}$ containing $\mathrm{I}$ i.e. $\varphi(\mathrm{z}) \in \mathrm{O}(\mathrm{U})$.For any complex neighbourhood $\mathrm{N}(\mathrm{I})$ containing $\mathrm{U}, \mathrm{B}(\mathrm{I})=\mathrm{O}(\mathrm{N}(\mathrm{I}) \backslash \mathrm{I}) \backslash \mathrm{A}(\mathrm{I})$, where $A(I)$ is the ring of all real analytic functions on $I$. Thus a hyper-function $\mathrm{f}(\mathrm{x}) \in \mathrm{B}(\mathrm{I})$ is determined by a defining function $\mathrm{F}(\mathrm{z})$, which is holomorphic in an adjacent(small) neighbourhood above and below the interval I, but is only determined up to a real analytic function on I.

For $\mathrm{F}(\mathrm{z})$, sometimes at $\mathrm{x} \in \mathrm{I}_{0} \subset \mathrm{I}$ the limit,

$\lim _{\varepsilon \rightarrow 0+}\left\{\mathrm{F}_{+}(\mathrm{x}+\mathrm{i} \varepsilon)-\mathrm{F}_{-}(\mathrm{x}-\mathrm{i} \varepsilon)\right\}$ exists. A point $\mathrm{x} \in \mathrm{I} \mathrm{II}_{0}$ is called a singular point and a point $\mathrm{x} \in \mathrm{I}_{0}$ is called a regular point of the hyper-function. At a regular point, a hyper-function $\mathrm{f}(\mathrm{x})$ has a function-value as an ordinary function. Hence $f(x)=F(x+i 0)-F(x-i 0)=\lim _{\varepsilon \rightarrow 0+}\left\{F_{+}(x+i \varepsilon)-F_{-}(x-i \varepsilon)\right\}$, provided the limit exists.

Definition 4[1]: A real analytic function $\varphi(\mathrm{x})$ is a function which is holomorphic in a full neighbourhood of the entire real axis i.e. $\varphi(x) \in A(R)$.

Definition 5[1]:The hyper-function $\mathrm{f}(\mathrm{x})=[\varphi(\mathrm{z})]$, where $\varphi(\mathrm{x}) \in \mathrm{A}(\mathrm{R})$ is any real analytic function, represents the 
zero hyper-function. We denote the zero hyper-function by 0 , since it can be identified with the ordinary zero function. Addition of two hyper-functions $\mathrm{f}(\mathrm{x}), \mathrm{g}(\mathrm{x}) \in \mathrm{B}(\mathrm{I})$ defined in the following way.

$$
\begin{aligned}
& \text { If } f(x)=[F(z)], g(x)=[G(z)], \text { define } \\
& f(x)+g(x)=[F(z)+G(z)] .
\end{aligned}
$$

Similarly scalar multiplication can be defined, for any

$\mathrm{c} \in \mathrm{C}, \operatorname{cf}(\mathrm{x})=\mathrm{c}[\mathrm{F}(\mathrm{z})]=[\mathrm{cF}(\mathrm{z})]$.

This addition and scalar multiplication are well define on $\mathrm{B}(\mathrm{I})$.

Proposition 6: B(I)is a linear space.

Definition 7[1]: If $f(x)=[F(z)] \in B(I)$ is a hyper-function and $\varphi(x) \in A(I)$ is a real analytic function on $I$,then $\varphi(x) f(x) \in B(I)$. Also $\varphi(x) f(x)=[\varphi(z) F(z)]$.

Definition 8[1]: For any given hyper-function

$f(x)=\left[F_{+}(z), F_{-}(z)\right]$ its derivative in the sense of hyperfunction is defined as

$$
\begin{gathered}
D f(x)=f(x)=\left[\frac{d F_{+}}{d z}, \frac{d F_{-}}{d z}\right] \\
D^{n} f(x)=f^{n}(x)=\left[\frac{d^{n} F_{+}}{d z^{n}}, \frac{d^{n} F_{-}}{d z^{n}}\right]
\end{gathered}
$$

Proposition 9: Hyper-functions are always infinitely differentiable.

Definition 10[1]: A hyper-function $\mathrm{f}(\mathrm{x})$ is called holomorphic at $x=a$, if the lower and upper component of the defining function can analytically be continued to a full(two- dimensional) neighborhood of the real point $a$ i.e. the upper/ lower component can analytically be continued across $a$ into the lower/upper half-plane.

Definition 11[1]: Let $\mathrm{f}(\mathrm{x})=\left[\mathrm{F}_{+}(\mathrm{z}), \mathrm{F}_{-}(\mathrm{z})\right]$ be a hyper-function, holomorphic at both endpoints of the finite interval $[\mathrm{a}, \mathrm{b}]$, then the (definite)integral of $f(x)$ over $[a, b]$ is defined and denoted by

$$
\int_{a}^{b} f(x) d x=\int_{\gamma_{a, b}^{+}} F_{+}(z) d z-\int_{\gamma_{a, b}^{-}} F_{-}(z) d z=-\oint_{(a, b)} F(z) d z
$$

where the contour $\gamma_{a, b}^{+}$runs in $N_{+}$from $a$ to $b$ above the real axis, and the contour $\gamma_{a, b}^{-}$is in $N_{\text {. from }} a$ to $b$ below the real axis.

Definition 12[1]: Consider hyper-functions depending on a continuous parameter $\alpha$ or an integral parameter $k$. The continuous parameter $\alpha$ varies in some open region of the complex plane and $\alpha_{0}$ is a limit point of . Integral parameter $k$ may vary in $N$ or $Z$. Then $f(x, \alpha)=$ $\left.[F(z, \alpha)], \alpha \in ; f_{k}(x)=\left[F_{k}(z)\right], k \in N o r Z\right\}$. We say that a family of holomorphic functions $F(z, \alpha)$, or a sequence of holomorphic functions $F_{k}(z)$ defined on a common domain $N \subset$ Cconverges uniformlyin the interior of $N$ to $F(z)$ as $\alpha \rightarrow \alpha_{0}$, or $k \rightarrow \infty$, respectively if $F(z, \alpha)$ or $F_{k}(z)$ converges uniformly to $F(z)$ in every compact sub domain of $N$. This uniform convergence in the interior of $N$ is also called compact convergence in $N$.

Definition 13[1]: Let $f(x)=\left[F_{+}(z), F_{-}(z)\right]$ be defined on $I$ such that, for every $k$, equivalent defining functions $G_{k}(z)$ of $F_{k}(z)$ exist, such that $G_{+k}(z)$ and $G_{-k}(z)$ are uniformly convergent in the interior of $N_{+}(I)$ and $N_{-}(I)$ to $F_{+}(z)$ and $F_{\text {- }}(z)$ respectively. Then we write $f(x)=\lim _{k \rightarrow \infty} f_{k}(x)$, and say that the sequence of hyper-functions $f_{k}(x)$ converges in the sense of hyper-functions to $f(x)$.

Proposition 14:If a limit in the sense of hyper-functions exists, it is unique.

Definition 15[1]: Let $f(x)$ is a hyper-function having a compact support $[a, b]$ with defining function $F(z) \in$ $O(N \backslash[a, b])$. Its upper and lower components are analytic continuations from each other. Such a hyper-function is called a perfect hyper-function.

Definition 16[1]: The hyper-function $f(x)=$ $\left[F_{+}(z), F_{-}(z)\right]$ is micro-analytic from above at $x=a \in I$, if the upper component $F_{+}(z)$ can analytically be continued across the real axis to a full neighbourhood of $a$. Similarly, $f(x)$ is micro-analytic from below at $x=a \in I$, if the lower component $F_{-}(z)$ can analytically be continued across the real axis to a full neighbourhood of $a$.

Definition 17[1]: Let $\sum_{0}$ be the largest open subset of the real line where the hyperfunction $f(x)=[F(z)]$ is vanishing. Its complement $K_{0}=R \backslash \sum_{0}$ is said to be the support of the hyperfunction $f(x)$ denoted by supp $f(x)$.

Let $\sum_{1}$ be the largest open subset of the real line where the hyperfunction $f(x)=[F(z)]$ is holomorphic. Its complement $K_{1}=R \backslash \sum_{1}$ is said to be the singular support of the hyperfunction $f(x)$ denoted by $\operatorname{singsupp} f(x)$.

Let $\sum_{2}$ be the largest open subset of the real line where the hyperfunction $f(x)=[F(z)]$ is micro-analytic. Its complement $K_{2}=R \backslash \sum_{2}$ is said to be the singular spectrum of the hyperfunction $f(x)$ denoted by sing spec $f(x)$.

Proposition 18[1]: For a hyper-function $\mathrm{f}(\mathrm{x})$ we have

$\operatorname{sing} \operatorname{specf}(\mathrm{x}) \subset \operatorname{sing} \operatorname{suppf}(\mathrm{x}) \subset \operatorname{suppf}(\mathrm{x})$

For the following let us consider open sets $J=(a, 0) \cup$ $(0, b)$ with some $a<0$ and some $b>0$ and compact subsets $K=\left[a^{\prime}, a^{\prime \prime}\right] \cup\left[b^{\prime}, b^{\prime \prime}\right]$ with $a<a^{\prime} \leq a^{\prime \prime}<0$ and $0<b^{\prime} \leq b^{\prime \prime}<b$. Also consider the following open neighbourhoods $[-\delta, \infty)+i J$ and $(-\infty, \delta]+i j$ of $R_{+}$ and $R_{-}$respectively for some $\delta>0$.

Consider the subclass $O\left(R_{+}\right)$of hyper-functions $f(x)=$ $[F(z)]$ on $R$ satisfying

(i) The support supp $f(x)$ is contained in $[0, \infty)$ 
(ii) Either the support $\operatorname{supp} f(x)$ is bounded on the right by a finite number $\beta>0$ or we demand that among all equivalent defining functions, there is one, $F(z)$ defined in $(-\infty, \delta]+i J$ such that for any compact $K \subset J$ there exist some real constant $M^{\prime}>0$ and $\sigma^{\prime}$ such that $|F(z)| \leq M^{\prime} e^{\sigma^{\prime} R z}$ holds uniformly for all $z \in[0, \infty)+i K$.

Because $\operatorname{supp} f(x) \subset R_{+}$and since the singular support singsuppf is a subset of the support, we have singsupp $f \subset R_{+}$. Therefore $f(x)$ is a holomorphic hyper-function for all $x<0$. Moreover, the fact that $F_{+}(x+i 0)-F_{-}(x-i 0)=0$ for all $x<0$ shows that $F(z)$ is real analytic on the negative part of the real axis. Hence $f(x) \in O\left(R_{+}\right)$implies that $\chi_{(-\varepsilon, \infty)} f(x)=f(x)$ for any $\varepsilon>0$.

Definition 19[1]: The subclass of hyper-functions $O\left(R_{+}\right)$is called the class of right sided originals.

In the case of an unbounded support $\operatorname{supp} f(x)$, let $\sigma_{-}=\inf \sigma^{\prime}$ be the greatest lower bound of all $\sigma^{\prime}$ where the infimum is taken over all $\sigma^{\prime}$ and all equivalent defining functions satisfying (ii). This number $\sigma_{-}=\sigma_{-}(f)$ is called the growth index of $f(x) \in O\left(R_{+}\right)$. It has the properties

(i) $\sigma_{-} \leq \sigma^{\prime}$

(ii) For every $\varepsilon>0$ there is a $\sigma^{\prime}$ with $\sigma_{-} \leq \sigma^{\prime} \leq \sigma_{-}+\varepsilon$ and an equivalent defining function $F(z)$ such that $|F(z)| \leq$ $M^{\prime} e^{\sigma^{\prime} R z}$ uniformly for all $z \in[0, \infty)+i K$.

In the case of a bounded support $\operatorname{supp} f(x)$,we set $\sigma_{-}(f)=$ $-\infty$.

Similarly, we introduce the class $O\left(R_{-}\right)$of hyper-functions specified by

1. The support $\operatorname{supp} f(x)$ is contained in $R_{-}=(-\infty, 0]$

2. Either the support $\operatorname{supp} f(x)$ is bounded on the left by a finite number $\alpha<0$, or we demand that among all equivalent defining functions there is one, denoted by $F(z)$ and defined in $(-\infty, \delta]+i J$ such that for any compact subset $K \subset J$ there are some real constants $M^{\prime \prime}>0$ and $|F(z)| \leq M^{\prime \prime} e^{\sigma{ }^{\prime \prime} R z}$ holds uniformly for $z \in(-\infty, 0]+i K$.

Definition 20[1]: The set $O\left(R_{-}\right)$is called the class of leftsided originals.

In the case of an unbounded support let $\sigma_{+}=\sup \sigma^{\prime \prime}$ be the least upper bound of all $\sigma^{\prime \prime}$, where the supremum is taken over all $\sigma^{\prime \prime}$ and all equivalent defining functions satisfying (ii). The number $\sigma_{+}=\sigma_{+}(f)$ is called the growth index of $f(x) \in O\left(R_{-}\right)$. It has the properties

(i) $\sigma^{\prime \prime} \leq \sigma_{+}$

(ii)For every $\varepsilon>0$ there is a $\sigma^{\prime \prime}$ such that $\sigma_{+}-\varepsilon \leq \sigma^{\prime \prime} \leq$ $\sigma_{+}$and a definig function $F(z)$ such that $|F(z)| \leq M^{\prime \prime} e^{\sigma^{\prime \prime} R z}$ uniformly for $z \in(-\infty, 0]+i K$

If the support $\operatorname{supp} f(x)$ is bounded, we set $\sigma_{+}(f)=+\infty$
With a left-sided original $g(x) \in O\left(R_{-}\right)$with growth index $\sigma_{+}(g)$ and a right-sided original $f(x) \in O\left(R_{+}\right)$with growth index $\sigma_{-}(f)$ form the hyper-function $h(x)=g(x)+h(x)$ whose support is now the entire real axis.

Definition 21[1]: Hyper-functions of the subclass $\mathrm{O}\left(R_{+}\right)$ are said to be of bounded exponential growth as $x \rightarrow \infty$ and hyper-functions of the subclass $\mathrm{O}\left(R_{-}\right)$are said to be of bounded exponential growth as $x \rightarrow-\infty$.

An ordinary function $f(x)$ is called of bounded exponential growth as $x \rightarrow \infty$, if there are some real constants $M^{\prime}>0$ and $\sigma^{\prime}$ such that $|f(x)| \leq M^{\prime} e^{\sigma^{\prime} x}$ for sufficiently large $x$. It is called of bounded exponential growth as $x \rightarrow-\infty$, if there are some real constants $M^{\prime \prime}>0$ and $\sigma^{\prime \prime}$ such that $|f(x)| \leq$ $M^{\prime \prime} e^{\sigma^{\prime \prime} x}$,for sufficiently negative large $x$.

A function or a hyper-function is of bounded exponential growth, if it is of bounded exponential growth for $x \rightarrow-\infty$ as well as for $\rightarrow \infty$.

\section{RESULTS}

Proposition 1:For a perfect hyper-function

$\mathrm{F}(\mathrm{x})=[\mathrm{F}(\mathrm{z})]$ in $\mathrm{B}(\mathrm{I})$,

$\operatorname{singspecf}(\mathrm{x})=\operatorname{singsupp}(\mathrm{x})=\operatorname{suppf}(\mathrm{x})$

As ordinary functions, hyper-functions also has the following properties.

Note: Here onwards we are considering a sub family of perfect hyper-functions having bounded exponential growth with compact support on $I \subset R$. We denote it by $\mathfrak{B}_{K}^{M}(I)$

Proposition 2: $\mathfrak{B}_{K}^{M}(I)$ is a linear subspace of $B(I)$

Definition 3: For $f(x)=[F(z)] \in \mathfrak{B}_{K}^{M}(I)$ the norm function $\|\|:. \mathfrak{B}_{K}^{M}(I) \rightarrow R$ is defined as

$\|f\|=\sup _{K \subset I}\{|f(x)|$

$=|F(x+i 0)-F(x-i 0)|:$ for all $x$

$\in K, K$ is a compact subset of I and for all $F(z)$

$\in[F(z)]\}$

Proposition 4: $\mathfrak{B}_{K}^{M}(I)$ is a normed linear space.

Definition 5: A sequence $f_{n}(x)=\left[F_{n}(z)\right]$ in $\mathfrak{B}_{K}^{M}(I)$ is a Cauchy sequence in $\mathfrak{B}_{K}^{M}(I)$ if for every $\varepsilon>0$ there exists $n_{0} \in N$ such that $\left\|f_{n}-f_{m}\right\|<\varepsilon$ whenever $n, m \geq n_{0}$.

Definition 6: A sequence $f_{n}(x)=\left[F_{n}(z)\right]$ in $\mathfrak{B}_{K}^{M}(I)$ is a convergent sequence in $\mathfrak{B}_{K}^{M}(I)$ if for every $\varepsilon>0$, $\left\|f_{n}-f\right\|$ $<\varepsilon$ as $n \rightarrow \infty$ and $f(x)=[F(z)]$ in $\mathfrak{B}_{K}^{M}(I)$

Proposition 7: $\mathfrak{V}_{K}^{M}(I)$ is a complete normed linear space. i.e. $\mathfrak{V}_{K}^{M}(I)$ is a Banach space

Proposition8: The set of all real valued hyper-functions $f(x)=[F(z)]$ in $\mathfrak{B}_{K}^{M}(I)$ is separable.

Proof: Set all hyper-function in $\mathfrak{B}_{K}^{M}(I)$ taking rational points values in $I \subset R$ will be a countable dense subset of $\mathfrak{V}_{K}^{M}(I)$ 
Definition 9: A hyper-function $\mathrm{f}(\mathrm{x})=[\mathrm{F}(\mathrm{z})] \in \mathfrak{B}_{K}^{M}(I)$ is called measurable if $F(z)$ is measurable.

Proposition 10: Let $\mathrm{f}(\mathrm{x})=[\mathrm{F}(\mathrm{z})], \mathrm{g}(\mathrm{x})=[\mathrm{G}(\mathrm{z})] \in \mathfrak{B}_{K}^{M}(I)$ are non-negative, real valued and measurable hyperfunctions. If $f(x) \leq g(x)$ then $\int f(x) d x \leq \int g(x) d x$

Proposition 11: Let $f_{k}(x)=\left[F_{k}(z)\right], k=1,2,3, \ldots$ be a sequence of hyper-functions in $\mathfrak{B}_{K}^{M}(I)$ and $f(x)=$ $\lim f_{k}(x)$, where $f(x)=[F(z)]$ then $\int\left|f_{k}(x)-f(x)\right| d x \rightarrow$ 0 as $k \rightarrow \infty$ in the sense of hyper-functions.

All the above propositions can be proved using the norm defined above and applying the existing results for the defining function of the exponentially bounded hyperfunctions.

\section{CONCLUSION}

Some characteristics of $\mathfrak{B}_{K}^{M}(I)$ are studied here. $\mathfrak{O}_{K}^{M}(I)$ is a Hilbert space if there exists a surjective linear isometry in the subspace of $\mathfrak{B}_{K}^{M}(I)$ having norm less than or equal to
1.The defined norm gave a normed space as well as metric space structure for $\mathfrak{B}_{K}^{M}(I)$. Also using the metric induced by the norm, a topology can be introduced in $\mathfrak{B}_{K}^{M}(I)$. Hence $\mathfrak{B}_{K}^{M}(I)$ is a topological space also.

\section{REFERENCES}

[1] U. Graf, Introduction to Hyper-functions and Their Integral Transforms, Birkhauser, 2010.

[2] S. Pilipovic and B. Stsnkovic, "Convergence in the space of Fourier Hyper-functions",Proc. Japan Acad. Ser. A Math. Sci., Vol. 73, No. 3, pp. 33-35,1997.

[3] B. Limaye, Functional Analysis, 3rd ed., New Delhi: New Age International India, 2014.

[4] G de Barra,Measure Theory and Integration, $2^{\text {nd }}$ ed., New Delhi:New Age International India,2013

[5] M. Sato, "Theory of hyper-functions I", J. Fac. Sci., Univ. Tokyo, Sect., Vol.1, pp. 139-193, 1959.

[6] M. Sato, "Theory of hyper-functions II", J. Fac. Sci., Univ. Tokyo, Sect., Vol.8, pp. 387-436,1960.

[7] M. Morimoto, Translations of Mathematical Monographs: An Introduction to Sato's Hyper-functions, American Mathematical Society, Providence, Rhode Island, 1993. 